\title{
Solving Einstein's equations for rotating spacetimes: Evolution of relativistic star clusters
}

\author{
Andrew M. Abrahams, ${ }^{*}$ Gregory B. Cook, ${ }^{*}$ Stuart L. Shapiro, ${ }^{\dagger}$ and Saul A. Teukolsky ${ }^{\ddagger}$ \\ Center for Radiophysics and Space Research, Cornell University, Ithaca, New York 14853
}

(Received 7 September 1993)

\begin{abstract}
A numerical relativity code designed to evolve rotating axisymmetric spacetimes is constructed. Both polarization states of gravitational radiation can be tracked. The source of the gravitational field is chosen to be a configuration of collisionless particles. The code is used to evaluate the stability of polytropic and toroidal star clusters. The formation of Kerr black holes by the collapse of unstable clusters is demonstrated. Unstable clusters with $J / M^{2}<1$ collapse to black holes, while those with $J / M^{2}>1$ collapse to new equilibrium configurations.

PACS number(s): 04.25.Dm, 04.20.Jb, 04.30.Db, 97.60.Lf
\end{abstract}

\section{INTRODUCTION}

A major effort is underway to develop computer codes that can solve Einstein's equations of general relativity for physically realistic systems. The spherical problem, where the field is nondynamical and no gravitational waves are produced, is essentially solved. The current focus is on methods for dealing with multidimensional systems with dynamical gravitational fields. While a number of important results have been obtained by numerical means, the field is beset by many technical difficulties. In addition to the usual problems of solving multidimensional partial differential equations numerically, relativity presents some unique complications. One of these is dealing with the appearance of singularities and black holes, which frequently form during gravitational collapse. The development of a singularity, for example, forces a numerical simulation to terminate. Another complication in numerical relativity is extracting information about gravitational waves when the wave amplitudes are typically much smaller than the background gravitational field variables. Because of these technical complications, developing and testing codes that solve Einstein's equations is a difficult enterprise.

So far, results from simulations using such codes have been presented for only a handful of nonspherical, asymptotically flat computations. These include calculations by Smarr [1], Eppley [2], Nakamura [3], Stark and Piran [4], Abrahams and Evans [5], the NCSA group [6], Shapiro and Teukolsky [7-9]. All of these calculations considered systems with axial symmetry. Of these, only those of Nakamura and Stark and Piran included rota-

\footnotetext{
*Also at the Center for Theory and Simulation in Science and Engineering and Laboratory of Nuclear Studies, Cornell University, Ithaca, NY.

${ }^{\dagger}$ Also at the Departments of Astronomy and Physics, Cornell University, Ithaca, NY.

${ }^{\ddagger}$ Also at the Departments of Physics and Astronomy, Cornell University, Ithaca, NY.
}

tion, for which both polarizations of the gravitational field are present. Both of these calculations treated fluid systems. In this paper we extend our earlier treatment [8] of axisymmetric systems with collisionless matter to include rotation.

The routines we construct to solve Einstein's field equations can be used for general axisymmetric spacetimes, including vacuum and fluid systems. Here we couple them to collisionless matter sources, for which the matter equations of motion are simply the geodesic equations and hence easy to integrate. The resulting code is useful for addressing a number of interesting questions that arise in the theory of relativistic stellar dynamics, as well as exploring the formation of black holes and the production of gravitational waves.

The exploration of nonspherical relativistic star clusters begins with a study of their equilibrium properties. Only recently have equilibrium models of such clusters been constructed. Shapiro and Teukolsky [10] have presented nonspherical models with no net rotation. Such models have equal numbers of corotating and counterrotating particles. They subsequently constructed models with net rotation [11]. These calculations could not address the dynamical stability of these equilibrium clusters, nor could they follow the fate of unstable systems. Such a study requires a truly dynamical code, and this is one of the principal applications of the code presented here.

The stability of spherical nonrotating clusters has been studied extensively (see Ref. [12] for a detailed discussion and references). For these clusters, the onset of dynamical instability occurs at the point of maximum binding energy along a sequence of increasing central density. Does this simple criterion carry over to rotating equilibria? Can rotation stabilize an unstable spherical cluster?

Collapse of an unstable rotating cluster to a Kerr black hole is another problem of considerable interest. Such collapse has never been treated for collisionless matter. Moreover, the earlier studies of axisymmetric rotating collapse of fluid stars [4] used a choice of coordinates that precluded the appearance of a black hole horizon. 
The coordinates used in our new code admit the presence of apparent horizons and so black hole formation can be diagnosed. Black holes that form are expected to settle down to Kerr holes at late times. Therefore, in principle, we can compare our numerically generated spacetime against Kerr as a check on the calculations.

Since it is impossible to form a Kerr black hole with angular momentum per unit mass $a / M>1$, the collapse of highly rotating configurations is an interesting topic of study. Such collapses in axisymmetry (where angular momentum cannot be radiated) must result in new stationary, nonsingular configurations, Kerr black holes surrounded by high angular-momentum matter, or naked singularities.

In Sec. II we present the basic equations we use. Also included in this section are several diagnostics for testing and calibrating the code, as well as an outline of the numerical scheme. In Sec. III we give results from representative simulations, including tests of gravitational wave propagation, studies of stability of rotating clusters, and collapse to Kerr black holes. Section IV summarizes our conclusions.

\section{EQUATIONS AND NUMERICAL ALGORITHM}

Here we discuss the coordinates and basic variables employed in our simulations. Gravitational units with $G=c=1$ are used throughout. We choose a rotating version of the quasi-isotropic spatial gauge along with the maximal time slicing condition. Many of the field equations in this gauge have been derived earlier in similar form [13] and the regularity and asymptotic properties of this gauge have also been previously investigated [13-15]. Here we give a consistent set of field equations in our notation. In the absence of rotation, these equations reduce to those presented in Ref. [9]. The spatial line element for an axisymmetric spacetime in quasi-isotropic gauge takes the following form in spherical-polar coordinates:

$$
d l^{2}=A^{2}\left(d r^{2}+r^{2} d \theta^{2}\right)+B^{2} r^{2}(\xi d \theta+\sin \theta d \phi)^{2},
$$

where the metric variables $A, B$, and $\xi$ are functions of $t$, $r, x \equiv \cos \theta$, but not $\phi$. It is useful to define the auxiliary variables

$$
\begin{aligned}
\varphi^{6} & =A^{2} B, \\
T & =\frac{A}{B}=e^{\eta}, \\
\psi & =e^{-\frac{\eta}{3}} \varphi=B^{1 / 2} .
\end{aligned}
$$

The variable $\eta$ represents the even-parity radiative component of the metric in the sense it approaches $h_{+}$at large distances from the source. The interpretation of $\xi$ is somewhat more complicated, but at large radii $\partial \xi / \partial r \rightarrow-\partial h_{\times} / \partial t$ assuming purely outgoing radiation $[14,15]$. Here $h_{+}$and $h_{\times}$are the asymptotic evenand odd-parity wave amplitudes.

The extrinsic curvature is projected on to the natural, rotating orthonormal basis yielding the components

$$
\begin{aligned}
A_{(1)(1)} & =A_{r}^{r}, \\
A_{(1)(2)} & =\frac{A_{\theta}^{r}}{r}-\xi \frac{A_{\phi}^{r}}{r \sin \theta}, \\
A_{(1)(3)} & =\frac{A}{B} \frac{A_{\phi}^{r}}{r \sin \theta}, \\
A_{(2)(3)} & =\frac{A}{B} \frac{A_{\phi}^{\theta}}{\sin \theta}, \\
A_{(3)(3)} & =A_{\phi}^{\phi}+\xi \frac{A_{\phi}^{\theta}}{\sin \theta}, \\
\lambda & \equiv A_{(1)(1)}+2 A_{(3)(3)},
\end{aligned}
$$

where the trace-free extrinsic curvature is defined as $A_{j}^{i}=K_{j}^{i}-\frac{1}{3} \delta_{j}^{i} K\left(K=K_{i}^{i}\right)$. It is interesting to note that, when written in terms of orthonormal components, the inclusion of rotation does not change the form of Eqs. (11), (12), (19), or (23) below, just the physical meaning of some of the terms. In the field equations we commonly use the conformally transformed components denoted with carets: $\hat{A}_{(i)(j)}=\varphi^{6} A_{(i)(j)}$.

\section{A. Gravitational field equations}

In the $3+1$ decomposition, Einstein's field equations break down into 4 coordinate conditions for the kinematical variables, 4 constraints, and 6 evolution equations each for the metric and extrinsic curvature components. Since a correct evolution of consistent initial data preserves the constraints, it is only necessary to use a subset of the constraint and evolution equations. In the fully constrained scheme used in this code, all four constraint equations are used: one to obtain a metric component and three to compute extrinsic curvature components. The remaining two metric and two extrinsic curvature components necessary in axisymmetry are evolved.

The shift vector components $\beta^{i}$ are determined on each slice by the requirement that the quasi-isotropic spatial gauge conditions be maintained in time. This results in a mixed parabolic-elliptic system of equations for the three components:

$$
\begin{aligned}
r \frac{\partial}{\partial r}\left(\frac{\beta^{r}}{r}\right)+\sqrt{1-x^{2}} \frac{\partial \beta^{\theta}}{\partial x} & =\frac{\alpha}{A^{2} B}\left(2 \hat{A}_{(1)(1)}+\hat{A}_{(3)(3)}\right) \\
r \frac{\partial \beta^{\theta}}{\partial r}-\sqrt{1-x^{2}} \frac{\partial}{\partial x}\left(\frac{\beta^{r}}{r}\right) & =2 \frac{\alpha}{A^{2} B} \hat{A}_{(1)(2)} \\
r \sqrt{1-x^{2}} \frac{\partial \beta^{\phi}}{\partial r}+r \xi \frac{\partial \beta^{\theta}}{\partial r} & =2 \frac{\alpha}{A B^{2}} \hat{A}_{(1)(3)}
\end{aligned}
$$

The lapse function $\alpha$, determining the temporal coordinate system, is found from the maximal slicing condition $K=\partial_{t} K=0$. This leads to the following linear elliptic equation, written in a Poisson-like form [16], which is solved on each time slice: 


$$
\begin{aligned}
\frac{1}{r^{2}} \frac{\partial}{\partial r}\left[r^{2} \frac{\partial}{\partial r}(\alpha \psi)\right]+\frac{1}{r^{2}} \frac{\partial}{\partial x}\left[\left(1-x^{2}\right) \frac{\partial}{\partial x}(\alpha \psi)\right]= & \frac{\alpha \psi}{4}\left[-\frac{1}{r} \frac{\partial}{\partial r}\left(r \frac{\partial \eta}{\partial r}\right)-\frac{\sqrt{1-x^{2}}}{r^{2}} \frac{\partial}{\partial x}\left(\sqrt{1-x^{2}} \frac{\partial \eta}{\partial x}\right)-\frac{B^{2}}{4 A^{2}}\left(\frac{\partial \xi}{\partial r}\right)^{2}\right. \\
& +\frac{7}{A^{2} B^{2}}\left\{\begin{array}{c}
\hat{\lambda}^{2}-3 \hat{\lambda} \hat{A}_{(3)(3)}+3 \hat{A}_{(3)(3)}^{2}+\hat{A}_{(1)(2)}^{2} \\
\left.\left.+\hat{A}_{(1)(3)}^{2}+\hat{A}_{(2)(3)}^{2}\right\}+8 \pi \frac{\rho^{*}+2 \hat{S}}{B}\right]
\end{array}\right.
\end{aligned}
$$

[See Sec. II B below for definitions of the source quantities $\rho^{*}, \hat{S}, \hat{S}^{i}$, and $\hat{S}_{i j}$.]

The extrinsic curvature components $\hat{A}_{(1)(1)}, \hat{A}_{(3)(3)}$, and $\hat{A}_{(1)(3)}$ are found by solving the momentum constraint equations on each slice:

$$
\begin{aligned}
& \frac{1}{r^{3}} \frac{\partial}{\partial r}\left(r^{3} \hat{A}_{(1)(1)}\right)+\hat{A}_{(3)(3)} \frac{\partial \eta}{\partial r}=8 \pi \hat{S}_{r}+\frac{1}{r} \frac{\partial}{\partial x}\left(\sqrt{1-x^{2}} \hat{A}_{(1)(2)}\right)+\frac{1}{T} \hat{A}_{(2)(3)} \frac{\partial \xi}{\partial r} \\
&-\frac{\partial}{\partial x}\left(\sqrt{1-x^{2}} \hat{A}_{(1)(1)}\right)- \frac{T}{\sqrt{1-x^{2}}} \frac{\partial}{\partial x}\left(\frac{\left(1-x^{2}\right)}{T} \hat{A}_{(3)(3)}\right) \\
&=-8 \pi \hat{S}_{\theta}+\frac{1}{r^{2}} \frac{\partial}{\partial r}\left(r^{3} \hat{A}_{(1)(2)}\right)+\frac{1}{r^{2}} \frac{\partial}{\partial r}\left(\frac{r^{3} \xi}{T} \hat{A}_{(1)(3)}\right)-\frac{\xi}{\sqrt{1-x^{2}}} \frac{\partial}{\partial x}\left(\frac{\left(1-x^{2}\right)}{T} \hat{A}_{(2)(3)}\right), \\
& \frac{\sqrt{1-x^{2}}}{r^{2}} \frac{\partial}{\partial r}\left(\frac{r^{3}}{T} \hat{A}_{(1)(3)}\right)=8 \pi \hat{S}_{\phi}+\frac{\partial}{\partial x}\left(\frac{\left(1-x^{2}\right)}{T} \hat{A}_{(2)(3)}\right) .
\end{aligned}
$$

The scalar Hamiltonian constraint, written in terms of the quantity $\psi$, takes the Poisson-like form

$$
\begin{aligned}
\frac{1}{r^{2}} \frac{\partial}{\partial r}\left[r^{2} \frac{\partial \psi}{\partial r}\right]+\frac{1}{r^{2}} \frac{\partial}{\partial x}\left[\left(1-x^{2}\right) \frac{\partial \psi}{\partial x}\right]= & -\frac{\psi}{4}\left[\frac{1}{r} \frac{\partial}{\partial r}\left(r \frac{\partial \eta}{\partial r}\right)+\frac{\sqrt{1-x^{2}}}{r^{2}} \frac{\partial}{\partial x}\left(\sqrt{1-x^{2}} \frac{\partial \eta}{\partial x}\right)\right. \\
& +\frac{1}{T^{2} \psi^{8}}\left\{\hat{\lambda}^{2}-3 \hat{\lambda} \hat{A}_{(3)(3)}+3 \hat{A}_{(3)(3)}^{2}+\hat{A}_{(1)(2)}^{2}+\hat{A}_{(1)(3)}^{2}+\hat{A}_{(2)(3)}^{2}\right\} \\
& \left.+\frac{1}{4 T^{2}}\left(\frac{\partial \xi}{\partial r}\right)^{2}\right]-\frac{2 \pi \rho^{*}}{\psi}
\end{aligned}
$$

The evolution equations for the "radiative" metric variables are

$$
\frac{\partial \eta}{\partial t}=\frac{\alpha}{A^{2} B} \hat{\lambda}+\beta^{r} \frac{\partial \eta}{\partial r}-\sqrt{1-x^{2}} \beta^{\theta} \frac{\partial \eta}{\partial x}-\left(1-x^{2}\right) \frac{\partial}{\partial x}\left(\frac{\beta^{\theta}}{\sqrt{1-x^{2}}}\right)
$$

and

$$
\frac{\partial \xi}{\partial t}=-2 \frac{\alpha}{A B^{2}} \hat{A}_{(2)(3)}+\beta^{r} \frac{\partial \xi}{\partial r}-\sqrt{1-x^{2}} \beta^{\theta} \frac{\partial \xi}{\partial x}-\left(1-x^{2}\right) \xi \frac{\partial}{\partial x}\left(\frac{\beta^{\theta}}{\sqrt{1-x^{2}}}\right)-\left(1-x^{2}\right) \frac{\partial \beta^{\phi}}{\partial x} .
$$

We note that, in contrast with Eq. (19), this last equation must be used in conjunction with the constraints for the establishment of correct initial data for a radiation-free, stationary configuration since $\hat{A}_{(2)(3)}$ can be nonzero even if $\xi=0$ :

$$
\frac{2 \alpha}{A B^{2}} \hat{A}_{(2)(3)}=-\left(1-x^{2}\right) \frac{\partial \beta^{\phi}}{\partial x} .
$$

Physically this can be justified by looking at a weak-field expansion; $\hat{A}_{(2)(3)}$ can include nonradiative current moments not present in $\xi$, e.g., a stationary octupole moment. (The condition $\eta=0$ does preclude the existence of a static quadrupole moment.) In practice, like Stark and Piran [4], rather than evolving $\xi$ itself we use $\partial \xi / \partial r$ as the principal, evolved variable and compute $\xi$ with a radial integration. This choice is made because the asymptotic expansion for $\xi$ includes an instantaneously propagated gauge term that does not fall off with radius $[13,15]$ making it more difficult to apply outgoing wave boundary conditions. Differentiating Eq. (20) with respect to $r$ and substituting Eq. (13) 
yields

$$
\begin{aligned}
\frac{\partial}{\partial t}\left[\frac{\partial \xi}{\partial r}\right]= & -2 \frac{\partial}{\partial r}\left(\frac{\alpha}{A B^{2}} \hat{A}_{(2)(3)}\right)-2\left(1-x^{2}\right) \frac{\partial}{\partial x}\left(\frac{\alpha}{r A B^{2}} \frac{\hat{A}_{(1)(3)}}{\sqrt{1-x^{2}}}\right)+\frac{\partial}{\partial r}\left(\beta^{r}\left[\frac{\partial \xi}{\partial r}\right]\right) \\
& -\left(1-x^{2}\right) \frac{\partial}{\partial x}\left(\frac{\beta^{\theta}}{\sqrt{1-x^{2}}}\left[\frac{\partial \xi}{\partial r}\right]\right) .
\end{aligned}
$$

The variable $\psi$ can also be evolved in order to provide an initial guess for Newton iteration of the nonlinear Hamiltonian constraint Eq. (18):

$$
\frac{\partial \psi}{\partial t}=\beta^{r} \frac{\partial \psi}{\partial r}-\sqrt{1-x^{2}} \beta^{\theta} \frac{\partial \psi}{\partial x}+\frac{\psi}{2}\left(\frac{\beta^{r}}{r}+\frac{x}{\sqrt{1-x^{2}}} \beta^{\theta}-\alpha \frac{\hat{A}_{(3)(3)}}{A^{2} B}\right) .
$$

Finally, extrinsic curvature components $\hat{A}_{(1)(2)}$ and $\hat{A}_{(2)(3)}$ are each evolved, corresponding, respectively, to the even- and odd-parity gravitational radiation degrees of freedom:

$$
\begin{aligned}
\frac{\partial \hat{A}_{(1)(2)}}{\partial t}= & \frac{1}{r^{3}} \frac{\partial}{\partial r}\left(r^{3} \beta^{r} \hat{A}_{(1)(2)}\right)-\frac{\partial}{\partial x}\left(\sqrt{1-x^{2}} \beta^{\theta} \hat{A}_{(1)(2)}\right) \\
& +\frac{\alpha A}{r^{2}}\left\{\frac{\partial}{\partial x}\left[\frac{\sqrt{1-x^{2}}}{A} \frac{\partial}{\partial r}(B r)\right]-\frac{1}{A^{2}} \frac{\partial}{\partial r}(A r) \frac{\partial}{\partial x}\left(B \sqrt{1-x^{2}}\right)\right\} \\
& -\hat{A}_{(1)(2)}\left(\sqrt{1-x^{2}} \frac{\partial \beta^{\theta}}{\partial x}+\frac{\partial \beta^{r}}{\partial r}\right)+\frac{2 \alpha \hat{A}_{(1)(3)} \hat{A}_{(2)(3)}}{A^{2} B} \\
& -\sqrt{1-x^{2}}\left(2 \hat{\lambda}-3 \hat{A}_{(3)(3)}\right) \frac{\partial}{\partial x}\left(\frac{\beta^{r}}{r}\right)+\frac{A B \sqrt{1-x^{2}}}{r} \frac{\partial}{\partial x}\left[\frac{1}{A} \frac{\partial \alpha}{\partial r}\right] \\
& -\frac{B \sqrt{1-x^{2}}}{A r^{2}} \frac{\partial}{\partial r}(A r) \frac{\partial \alpha}{\partial x}-\frac{8 \pi \alpha}{A^{2} r}\left(\hat{S}_{r \theta}-\frac{\xi}{\sqrt{1-x^{2}}} \hat{S}_{r \phi}\right)
\end{aligned}
$$

and

$$
\begin{aligned}
\frac{\partial \hat{A}_{(2)(3)}}{\partial t}= & \frac{1}{r^{2}} \frac{\partial}{\partial r}\left(r^{2} \beta^{r} \hat{A}_{(2)(3)}\right)-\frac{\partial}{\partial x}\left(\sqrt{1-x^{2}} \beta^{\theta} \hat{A}_{(2)(3)}\right) \\
& -\frac{A}{2 B r^{2}} \frac{\partial}{\partial r}\left(\frac{B^{3} r^{2} \alpha}{A^{2}} \frac{\partial \xi}{\partial r}\right)-r \hat{A}_{(1)(3)} \frac{\partial \beta^{\theta}}{\partial r}+\frac{\alpha \hat{\lambda} \hat{A}_{(2)(3)}}{A^{2} B}-\frac{8 \pi \alpha}{A B r^{2} \sqrt{1-x^{2}}}\left(\hat{S}_{\theta \phi}-\frac{\xi}{\sqrt{1-x^{2}}} \hat{S}_{\phi \phi}\right) .
\end{aligned}
$$

In Sec. IID we discuss the order of solution and the differencing techniques used in numerical implementation of this system of equations.

\section{B. Matter evolution}

The geodesic equations for the motion of the collisionless matter particles are written in first order form as

$$
\frac{d r}{d t}=\frac{\alpha u_{r}}{A^{2} \hat{u}}-\beta^{r}, \frac{d \theta}{d t}=\frac{\alpha u_{\theta}}{r^{2} A^{2} \hat{u}}-\frac{\alpha \xi u_{\phi}}{r^{2} \sin \theta A^{2} \hat{u}}-\beta^{\theta}, \frac{d \phi}{d t}=\frac{\alpha\left(A^{2}+\xi^{2} B^{2}\right) u_{\phi}}{r^{2} \sin ^{2} \theta A^{2} B^{2} \hat{u}}-\frac{\alpha \xi u_{\theta}}{r^{2} \sin \theta A^{2} \hat{u}}-\beta^{\phi},
$$

and

$$
\begin{aligned}
\frac{d u_{r}}{d t}= & -\hat{u} \frac{\partial \alpha}{\partial r}+u_{r} \frac{\partial \beta^{r}}{\partial r}+u_{\theta} \frac{\partial \beta^{\theta}}{\partial r}+u_{\phi} \frac{\partial \beta^{\phi}}{\partial r} \\
& -\frac{\alpha}{2 \hat{u}}\left\{\frac{\partial}{\partial r}\left(\frac{1}{A^{2}}\right) u_{r}^{2}+\frac{\partial}{\partial r}\left(\frac{1}{r^{2} A^{2}}\right) u_{\theta}^{2}+\frac{\partial}{\partial r}\left(\frac{A^{2}+\xi^{2} B^{2}}{r^{2} \sin ^{2} \theta A^{2} B^{2}}\right) u_{\phi}^{2}-2 \frac{\partial}{\partial r}\left(\frac{\xi}{r^{2} \sin \theta A^{2}}\right) u_{\theta} u_{\phi}\right\}, \\
\frac{d u_{\theta}}{d t}= & -\hat{u} \frac{\partial \alpha}{\partial \theta}+u_{r} \frac{\partial \beta^{r}}{\partial \theta}+u_{\theta} \frac{\partial \beta^{\theta}}{\partial \theta}+u_{\phi} \frac{\partial \beta^{\phi}}{\partial \theta} \\
& -\frac{\alpha}{2 \hat{u}}\left\{\frac{\partial}{\partial \theta}\left(\frac{1}{A^{2}}\right) u_{r}^{2}+\frac{\partial}{\partial \theta}\left(\frac{1}{r^{2} A^{2}}\right) u_{\theta}^{2}+\frac{\partial}{\partial \theta}\left(\frac{A^{2}+\xi^{2} B^{2}}{r^{2} \sin ^{2} \theta A^{2} B^{2}}\right) u_{\phi}^{2}-2 \frac{\partial}{\partial \theta}\left(\frac{\xi}{r^{2} \sin \theta A^{2}}\right) u_{\theta} u_{\phi}\right\}, \\
\frac{d u_{\phi}}{d t}= & 0 .
\end{aligned}
$$


The quantity $\hat{u}$ is defined via the normalization condition for the four-velocity:

$$
\begin{aligned}
\hat{u} \equiv \alpha u^{t}= & {\left[1+\frac{u_{r}^{2}}{A^{2}}+\frac{u_{\theta}^{2}}{r^{2} A^{2}}+\frac{\left(A^{2}+\xi^{2} B^{2}\right) u_{\phi}^{2}}{r^{2} \sin ^{2} \theta A^{2} B^{2}}\right.} \\
& \left.-2 \xi \frac{u_{\theta} u_{\phi}}{r^{2} \sin \theta A^{2}}\right]^{\frac{1}{2}} .
\end{aligned}
$$

Note that the particles can rotate $\left(u_{\phi}\right.$ can be nonzero) but in axisymmetry the angular momentum of each particle is conserved; i.e., $u_{\phi}$ is constant in time.

The matter source terms are found by first binning the particles (each of mass $m$ ), and then computing the sums

$$
\begin{aligned}
& \rho^{*}=\sum_{k} \frac{m(\hat{u})_{k}}{\left(r^{2} \sin \theta d r d \theta d \phi\right)_{k}} \\
& \hat{S}_{i}=\sum_{k} \frac{m\left(u_{i}\right)_{k}}{\left(r^{2} \sin \theta d r d \theta d \phi\right)_{k}},
\end{aligned}
$$

$$
\begin{aligned}
\hat{S}_{i j} & =\sum_{k} \frac{m\left(u_{i}\right)_{k}\left(u_{j}\right)_{k}}{(\hat{u})_{k}\left(r^{2} \sin \theta d r d \theta d \phi\right)_{k}} \\
\hat{S} & =\rho^{*}-\sum_{k} \frac{m}{(\hat{u})_{k}\left(r^{2} \sin \theta d r d \theta d \phi\right)_{k}} .
\end{aligned}
$$

These quantities are conformal rescalings of the standard Arnowitt-Deser-Misner (ADM) stress-energy tensor projections [16]: $\rho^{*}=\varphi^{6} \rho, \hat{S}_{i}=\varphi^{6} S_{i}$, and $\hat{S}_{i j}=\varphi^{6} S_{i j}$.

\section{Diagnostics}

During the course of numerical evolution, a set of physical diagnostics are computed which comprise the principal checks on the reliability of our dynamical calculations in the nonlinear regime. We compute the mass and angular momentum of the spatial hypersurfaces and look for trapped regions. If we choose a two-sphere in the asymptotic weak-field regime, surrounding a source of gravitational radiation, the Brill mass of the spatial slice may be computed by the expression

$$
\begin{aligned}
M_{B}=-\frac{1}{2 \pi} \int d V \Delta \ln \psi=\frac{1}{2 \pi} \int d V & {\left[(\nabla \ln \psi)^{2}+\frac{1}{4 T^{2} \psi^{8}}\left\{\hat{\lambda}^{2}-3 \hat{\lambda} \hat{A}_{(3)(3)}+3 \hat{A}_{(3)(3)}^{2}+\hat{A}_{(1)(2)}^{2}+\hat{A}_{(1)(3)}^{2}+\hat{A}_{(2)(3)}^{2}\right\}\right.} \\
& \left.+\frac{1}{16 T^{2}}\left(\frac{\partial \xi}{\partial r}\right)^{2}+\frac{2 \pi \rho^{*}}{\psi^{2}}\right]
\end{aligned}
$$

where the integral is taken over the interior coordinatevolume and $\Delta$ and $\nabla$ denote the three-dimensional, flat-space Laplacian and gradient operators, respectively. The Brill mass, corrected for the mass flux in the form of gravitational radiation that leaves the grid during the simulation, is a conserved quantity. The radiated mass energy is approximated by

$$
\triangle M_{B}=\frac{1}{16 \pi} \int d t \int d \Omega r^{2} A B\left(\lambda^{2}+4 A_{(2)(3)}^{2}\right)
$$

The mass $M_{B}$ is compared against an estimate of the ADM mass read off from the $1 / r$ term in the conformal factor $\phi$.

Since in axisymmetry gravitational radiation carries no angular momentum, the total angular momentum of the collisionless matter system is another conserved quantity. We compute the angular momentum via two independent expressions. From the definition of angular momentum in an axisymmetric spacetime, we have a surface integral expression valid in the vacuum exterior:

$$
\begin{aligned}
J & =\frac{1}{8 \pi} \int d S_{i}\left(\frac{\partial}{\partial \phi}\right)^{j} A_{j}^{i} \\
& =\frac{r^{3}}{2} \int_{0}^{1} d x\left(1-x^{2}\right)^{1 / 2} \frac{\hat{A}_{(1)(3)}}{T} .
\end{aligned}
$$

The alternative expression is a volume integral over the rotating matter:

$$
\begin{aligned}
J & =\int d V S_{i}\left(\frac{\partial}{\partial \phi}\right)^{i} \\
& =4 \pi \int_{0}^{R} d r \int_{0}^{1} d x r^{2} \hat{S}_{\phi}
\end{aligned}
$$

This is equivalent to summing the individual angular momenta of all the elements. Thus, this expression is automatically conserved as $u_{\phi}$ is constant [see Eq. (27)]. However its conservation in the code is a test of how well the particle binning algorithm (which produces $\hat{S}_{\phi}$ from $u_{\phi}$ ) represents the actual particle distribution.

It should be noted that $J$ cannot be found simply by looking at the asymptotic behavior of the shift vector component $\beta^{\phi}$ for nonstationary spacetimes. The near origin behavior of $\beta^{\phi}$ is determined only up to a possibly angle-dependent constant of integration [see Eq. (13)]. Correcting the dipole part of the shift vector to give the usual post-Newtonian behavior $\beta^{\phi} \rightarrow-2 J / r^{3}$ could be done as postprocessing but requires knowledge of $J$ and results in a slight irregularity in the form of angular dependence at the origin.

A final, crucial probe in our study of black hole formation is the apparent horizon solver. It turns out that in the orthonormal basis, the apparent horizon equation is unchanged from the nonrotating case, though the physical meaning of some of the extrinsic curvature components is changed. Thus, the same equations and algorithm described in Ref. [9] can be employed. 


\section{Numerical method}

Here we give a brief discussion of our numerical method; more details about the code in its nonrotating form may be found in Ref. [9]. The spatial derivatives appearing in the equations of Sec. II are differenced using standard second-order methods for uniform grids. All time evolutions are performed with a leapfrog scheme.
We choose code variables based on regularity considerations. The regular behavior of variables at the origin was originally worked out by Bardeen and Piran [13] and is summarized in the Appendix. For instance, $\hat{A}_{(1)(2)} / \sin \theta$ and $\hat{A}_{(2)(3)} / \sin ^{2} \theta$ are the evolved components of the extrinsic curvature. Our spatial differencing also pays close attention to the regularity. For instance, the evolution equation (25) for the $\hat{A}_{(2)(3)}$ is rewritten as

$$
\begin{aligned}
\frac{\partial}{\partial t}\left[\frac{\hat{A}_{(2)(3)}}{\sin ^{2} \theta}\right]= & 4 r \beta^{r} \frac{\partial}{\partial r^{4}}\left(r^{2}\left[\frac{\hat{A}_{(2)(3)}}{\sin ^{2} \theta}\right]\right)-\left(1-x^{2}\right)\left[\frac{\beta^{\theta}}{\sin \theta}\right] \frac{\partial}{\partial x}\left[\frac{\hat{A}_{(2)(3)}}{\sin ^{2} \theta}\right] \\
& \left.+\left[\frac{\hat{A}_{(2)(3)}}{\sin ^{2} \theta}\right] \frac{\partial \beta^{r}}{\partial r}-\left(1-x^{2}\right)\left[\frac{\hat{A}_{(2)(3)}}{\sin ^{2} \theta}\right] \frac{\partial}{\partial x}\left[\frac{\beta^{\theta}}{\sin \theta}\right]-2 r^{2}\left[\frac{\hat{A}_{(1)(3)}}{\sin \theta}\right] \frac{\partial}{\partial r^{2}}\left[\frac{\beta^{\theta}}{\sin \theta}\right]+4 x\right] \frac{\hat{A}_{(2)(3)}}{\sin { }^{2} \theta}\left[\frac{\beta^{\theta}}{\sin \theta}\right] \\
& -\frac{3 r^{2} \alpha}{2} \frac{B^{2}}{A} \frac{\partial}{\partial r^{3}}\left[\frac{\partial_{r} \xi}{\sin ^{2} \theta}\right]-3 r \alpha \frac{B}{A} \frac{\partial B}{\partial r^{2}}\left[\frac{\partial_{r} \xi}{\sin ^{2} \theta}\right]-\frac{\alpha}{r} \frac{B^{2}}{A}\left[\frac{\partial_{r} \xi}{\sin ^{2} \theta}\right]-r \frac{B^{2}}{A} \frac{\partial \alpha}{\partial r^{2}}\left[\frac{\partial_{r} \xi}{\sin ^{2} \theta}\right] \\
& +2 r \alpha \frac{B^{2}}{A^{2}} \frac{\partial A}{\partial r^{2}}\left[\frac{\partial_{r} \xi}{\sin ^{2} \theta}\right]+\frac{\alpha \hat{\lambda}}{A^{2} B}\left[\frac{\hat{A}_{(2)(3)}}{\sin ^{2} \theta}\right]-8 \pi \alpha\left[\frac{\hat{S}_{(2)(3)}}{\sin ^{2} \theta}\right]
\end{aligned}
$$

The order of solution for the field and matter equations is as follows. First the coordinate velocities and the accelerations of the particles, the right-hand sides of Eqs. (26) and (27), are calculated. Then the metric, extrinsic curvature and particles are evolved forward in time. Next the matter density and stresses are recalculated and used in the solution of the constraint equations. To solve the momentum constraint system, first the "rotation" momentum constraint Eq. (17) is integrated, yielding $\hat{A}_{(1)(3)}$ which in turn is used as a source in the integration of Eqs. (15) and (16). The evolved variables are then used as sources for the Hamiltonian constraint, lapse, and shift equations. To compute the shift vector, the elliptic subsystem for $\beta^{r}$ and $\beta^{\theta}$ is solved first followed by the parabolic integration for $\beta^{\phi}$.

In order to maintain resolution of both particles and fields the radial and angular mesh locations are allowed to move in time. Specifically, a fraction of the radial mesh (typically more than one half) is constrained to be in the source region. In toroidal cases (see Sec. III) the mesh is most concentrated at the outer edge of the matter. The angular mesh is also allowed to fan in time to track the matter.

There are a number of subtleties involved in finite differencing the field equations. For example, the even parity quantity $\eta$ goes like $r^{2}$ near the origin, and enforcing this via Eq. (19) requires a delicate numerical cancellation on the right-hand side. Similarly, the regularity requirements of the metric evolution equation (22) must be addressed. The quantity $\partial \xi / \partial r$ has an $r^{3}$ dependence near the origin while $\partial \hat{A}_{(2)(3)} / \partial r$ and $\hat{A}_{(1)(3)} / r$ each go like $r$ (see the Appendix). This incompatibility (owing to axisymmetry) requires a delicate cancellation in Eq. (22) if regularity of $\xi$ is to be maintained. This cancellation is enforced numerically via the momentum constraint Eq. (17). The coefficient of the leading order, $r^{2}$ part of $\hat{A}_{(2)(3)}$ (known from evolution) is calculated near the origin and used to set the value of $\hat{A}_{(1)(3)}$ on the first radial zone, improving enormously the cancellation of the leading order radial parts of these quantities near the origin. This process reduces the error that would otherwise drive an instability near the origin. Finally, to further control the growth of unstable modes, we add Kreiss-Oliger [17] dissipation terms to the evolution equations for both the even- and odd-parity metric and extrinsic curvature variables. Our experience has been that the odd-parity equations require both angular and radial dissipation while for the even-parity parts of the field it is sufficient to add only radial dissipation. For the angular dissipation, we use a second-order derivative operator on the field quantity; a fourth-order operator is used for radial dissipation. In each case the operators are constructed to preserve regularity of the evolved quantity.

\section{RESULTS}

In this section we present various numerical results obtained with the rotating code. Except when noted otherwise, the computational mesh consisted of 200 radial and 16 angular zones (for one quadrant) and 3000 particles were used. Verification runs were performed in several cases with 32 angular zones and 6000 or 12000 particles. The outer boundary of the mesh is placed at $50 \mathrm{M}$, well outside the edge of the matter distribution. Typical run durations were $\sim 2 \times 10^{4}$ time steps although in some cases of stability evaluation the runs were several times longer. For the runs discussed here, the mass indicators were conserved, and consistent, at a tolerance level of a few percent. Calculations were performed on IBM ES9000 and RS/6000 computers. A typical run with these resources took $10 \mathrm{CPU}$ hours on the ES-9000.

A number of vacuum test runs were carried out, pri- 


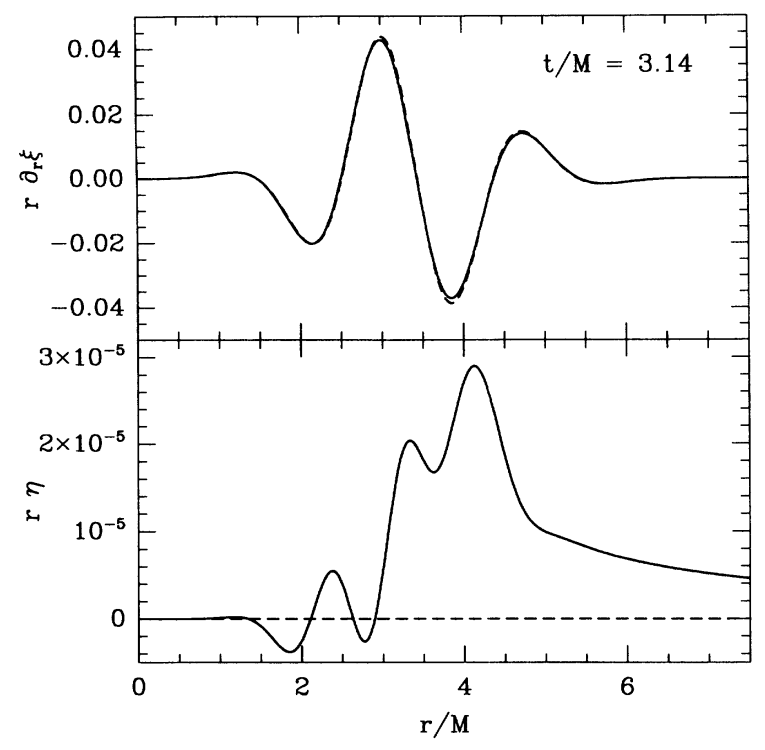

FIG. 1. Comparison between analytic (dashed line) and numerical (solid line) solutions for a linearized odd-parity wave propagating in vacuum. The upper frame shows a snapshot of the odd parity variable $\partial_{r} \xi$ times the radius $r$ as a function of $r / M$. The lower frame shows the small even-parity wave produced in $\eta$ by nonlinear interactions.

marily designed to evaluate the code's ability to propagate gravitational waves of both polarizations. These runs led to the refinement of our algorithm for the oddparity constrained evolution system as described in the last section. A linearized, quadrupole time-symmetric wave with either or both polarizations is established at the origin (see Refs. $[18,15])$. The even-parity wave has quadrupolar and the odd-parity wave octupolar angular dependence (even- $\ell$ odd-parity waves do not exist in equatorial plane symmetry). We evolved such waves forward in time and found excellent agreement with the analytic solution from linearized theory once the odd-parity instability was controlled. Results from a propagation test with an odd-parity wave is shown in Fig. 1. Note that the even-parity wave appearing in $\eta$ arises from the small nonlinear self-interaction of the wave.

\section{A. Dynamical stability of rotating clusters}

We considered two types of collisionless matter configurations: polytropic and toroidal. In Table I, we give a complete list of the cases considered in this paper, including the type of configuration, initial compactness, angular momentum, and final state. First we describe the use of the code to study the dynamical stability of equilibrium models.

The details of the construction of equilibrium initial data for these cases can be found in Ref. [11]. To summarize, the particle distributions are specified by choosing a phase space distribution function for the matter in the form

$$
f\left(E, J_{z}\right)=g(E) h\left(J_{z}\right)
$$

where $E=-u_{0}$ is the particle energy and $J_{z}=u_{\phi}$ is the angular momentum, both per unit mass. For the polytropic configurations, the energy distribution takes the form

$$
g(E)=\left\{\begin{array}{l}
K\left[\left(\frac{E}{E_{\max }}\right)^{2}\right]^{-\delta}\left[1-\left(\frac{E}{E_{\max }}\right)^{2}\right]^{\delta}, \quad E \leq E_{\max } \\
0, \quad E>E_{\max },
\end{array}\right.
$$

where $K$ and $E_{\max }$ are constants and $\delta$ is related to the polytropic index $n$ by $n=\delta+3 / 2$. The adopted angular momentum distribution has the Gaussian form

$$
h\left(J_{z}\right)=\frac{1}{\pi^{1 / 2} J_{0}} \exp \left(\mp J_{z}^{2} / J_{0}^{2}\right)
$$

TABLE I. Fates of selected collisionless matter configurations.

\begin{tabular}{l|c|c|c|c}
\hline \hline Case $^{\mathrm{a}}$ & Initial model $^{\mathrm{b}}$ & $R_{s} / M$ & $J / M^{2}$ & Outcome \\
\hline 1 & Polytrope & 12.0 & 0.00 & Stable \\
2 & Polytrope & 8.0 & 0.00 & Collapse to Schwarzschild \\
$3^{*}$ & Polytrope & 11.0 & 0.61 & Stable \\
$4^{*}$ & Polytrope & 11.0 & 0.94 & Stable \\
$5^{*}$ & Polytrope & 8.0 & 0.55 & Collapse to Kerr \\
$6^{*}$ & Polytrope & 8.0 & 0.95 & Stable \\
$7^{*}$ & Polytrope & 8.0 & 1.75 & Stable \\
8 & Equilibrium toroid & 7.0 & 0.0 & Stable \\
9 & Equilibrium toroid & 7.0 & 1.07 & Stable \\
10 & Toroid, cut down 0.5 & 4.5 & 0.65 & Collapse to Kerr \\
11 & Toroid, cut down 0.65 & 4.5 & 0.89 & Collapse to Kerr \\
12 & Toroid, cut down 0.72 & 4.5 & 0.98 & Collapse to Kerr \\
13 & Toroid, cut down 0.80 & 4.5 & 1.05 & Collapse to new equilibrium \\
14 & Toroid, cut down 0.90 & 4.5 & 1.18 & Collapse to new equilibrium \\
$15^{*}$ & Equilibrium toroid & 4.5 & 1.34 & Stable \\
\hline \hline
\end{tabular}

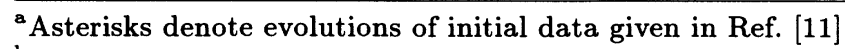

${ }^{b}$ All polytropes have index $n=1.5$ and are equilibrium configurations constructed from Eqs. (38)(41). Equilibrium toroids are constructed from Eqs. (38), (42), and (43). In cases 10-14 the $u_{\phi}$ velocities have been cut down by the indicated factor. 
where $J_{0} \rightarrow \infty$ gives a spherical configuration, since the distribution function is then independent of angular momentum. A positive sign in the exponential leads to oblate clusters, while a negative sign produces prolate clusters.

Toroidal clusters are produced with $\delta$ function distributions:

$$
\begin{aligned}
g(E) & =K \delta\left(E-E_{\max }\right) \\
h(J) & =\frac{1}{2}\left[\delta\left(J_{z}-J_{0}\right)+\delta\left(J_{z} \pm J_{0}\right)\right] .
\end{aligned}
$$

Here a positive sign in the angular momentum distribution function leads to equal numbers of corotating and counterrotating particles and no net angular momentum. A minus sign leads to net rotation for the configuration, with $J=\left(M_{0} / m\right) J_{0}$, where $M_{0}$ is the total rest mass of the configuration and $m$ is the rest mass of one of the individual (identical) particles. For both types of distributions, $E_{\max }$ is chosen to correspond to the maximum energy of a particle in a spherical cluster parameterized by radius $R_{s}$ (in Schwarzschild coordinates) yielding $E_{\max }=\left(1-R / R_{s}\right)^{1 / 2}$.

The field equations (assuming a stationary, axisymmetric metric) are solved (see Ref. [11]) yielding the metric quantities $A$ and $B$ ( $\eta$ is nonzero) and the extrinsic curvature components $\hat{A}_{(1)(3)}$ and $\hat{A}_{(2)(3)}[19]$. The metric function $\xi$ and the other extrinsic curvature components are all zero initially. Particle data in the form of positions and four-velocities are found by random samplings of the distribution functions.

The code was first run for a nonrotating spherical polytrope with index $n=1.5$ to compare against the expectations in Ref. [11]. The case with $R_{s} / M=12$ (case 1 ) was stable as predicted, because it resides on the stable side of the maximum in the binding energy curve, which occurs at $R_{s} / M \sim 9$. Run 2 with $R_{s} / M=8$ was unstable and collapsed to a Schwarzschild black hole. The apparent horizon formed at a time of about $82 M$. Next we consider a polytrope with $J_{0} \rightarrow \infty$ so that the distribution is identical to the spherical model, except that now all particles are made to rotate in the same sense. As a result, the cluster is nonspherical and has net angular momentum. In case $3 R_{s} / M=11$ and $J / M^{2}=0.61$. We evolved this cluster for a time greater than $100 M$ with no indication of instability. Even when the angular momentum was increased to $J / M^{2}=0.94$ by setting $J_{0}=0.4$ (case 4 ) the configuration was still stable. This configuration was evolved for a time $\sim 140 \mathrm{M}$.

Making all the particles rotate with the same sense in a configuration on the unstable branch of the binding energy curve does not provide enough angular momentum to prevent collapse. Such a polytrope with $R_{s} / M=8$ and $J_{0} \rightarrow \infty$ (case 5$)$ collapsed to a Kerr black hole. In Fig. 2 we compare this collapse to the nonrotating spherical collapse from the same initial radius (case 2 ). Increasing the angular momentum sufficiently does lead to stabilization. We verified this in case $6\left(J / M^{2}=0.95\right)$ and case $7\left(J / M^{2}=1.75\right)$. As shown in Fig. $5(\mathrm{~d})$ of Ref. [11] this latter configuration is very flattened and has a very low density in the center. It is not dissimilar to the toroidal configurations discussed below.

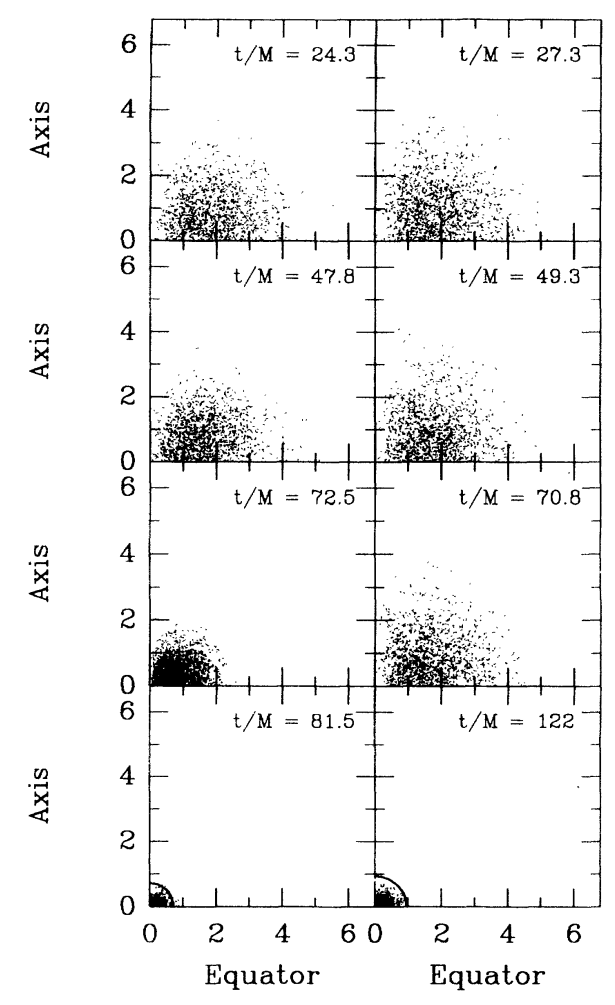

FIG. 2. Comparison of collapses of unstable polytropes. The left column shows snapshots from the collapse of a spherical nonrotating polytrope to a Schwarzschild black hole (case 2 ). This polytrope has equal numbers of corotating and counterrotating particles so that there is no net rotation. The right column shows the collapse of the analogous polytrope (case 5) constructed by making all the particles rotate with the same sense. While the evolution would be identical in Newtonian theory, the collapse of the rotating polytrope eventually leads to a Kerr black hole. An apparent horizon (solid line) forms in the nonrotating case at $t \sim 80 M$ and at $t \sim 120 M$ in the rotating case. Each frame shows a $\phi=$ const slice of the axisymmetric solution.

In the numerical evolution of a stable configuration, small perturbations in the initial data induced by numerical inaccuracies lead to oscillations and the generation of gravitational waves. For example, consider the most relativistic configuration, case 15 , a toroid with $R_{s} / M=4.5$. In Fig. 3 we plot an estimate of the evenparity gravitational waveform $h_{+}$(extracted using methods of Ref. [15]) as a function of time and see a distinct pulse at $t \sim 20 M$ followed by some further oscillations. Also plotted in Fig. 3 is a measure of how the "mean height" $\bar{Z}$ of the toroid above the equatorial symmetry plane differs from its initial value. With the waveform being measured at a radius of $10 M$, we believe that there is a convincing correlation between the main wave pulse and the oscillation in the minor radius of the toroid (analogous to the radius of the circle obtained by taking a cross section of a toroid) There is also an oscillation in the major radius of the toroid (analogous to the radius from the axis of rotation to the center of the circle which, when revolved, creates the toroid) as we will see later in 


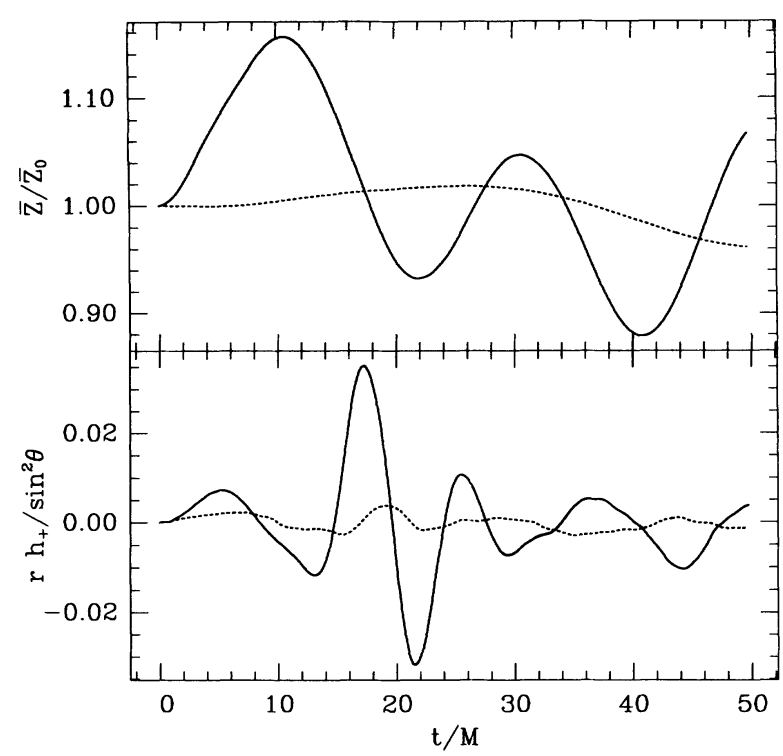

FIG. 3. Correlation of oscillations in the mean height $\bar{Z}$ of a toroid with the $h_{+}$waveform for cases 9 (dashed lines) and 15 (solid lines). In the upper frame, we plot the ratio of $\bar{Z}$ to its initial value $\bar{Z}_{0}$. In the lower frame, we plot $h_{+}$as measured at $r=10 \mathrm{M}$. Note that case 15 undergoes larger oscillations in $\bar{Z}$ than does case 9 and this leads to a larger amplitude for $h_{+}$.

Fig. 8. We also plot, in Fig. 3, results from the less relativistic case 9 , with $R_{s} / M=7$. This case appears much more stable as measured by the fractional height oscillation and the emitted wave. If these cases are truly stable, stationary equilibrium configurations, then reducing the numerical error by increasing computational resources should reduce the oscillations of the toroids along with their gravitational radiation. Even with the modest resources employed here, we can conclude that all the equilibrium toroidal configurations studied in this paper are stable against collapse to a black hole.

\section{B. Collapse of toroidal clusters}

In order for the toroidal configurations to collapse to black holes it is necessary to produce nonequilibrium initial data, because all the equilibrium cases were found to be stable. To accomplish this we "cut down" the equilibrium value of $u_{\phi}$ for each particle, thereby reducing its angular momentum and the total angular momentum of the system. The constraints are then iterated to obtain consistent initial data.

In case $10, u_{\phi}$ was cut down by a factor of 0.5 , reducing the angular momentum of the system to $J / M^{2}=$ 0.65. We show the evolution of the particle distribution in Fig. 4. Initially the toroid collapses along its minor radius to a thin hoop. Then, while undergoing oscillations along the minor radius, it collapses inward along the major radius. As the major radius passes through a quasi-isotropic value of about $0.5 \mathrm{M}$ the apparent horizon forms at a time of about $18 \mathrm{M}$. As seen in Fig. 5, the horizon grows as the collapse progresses

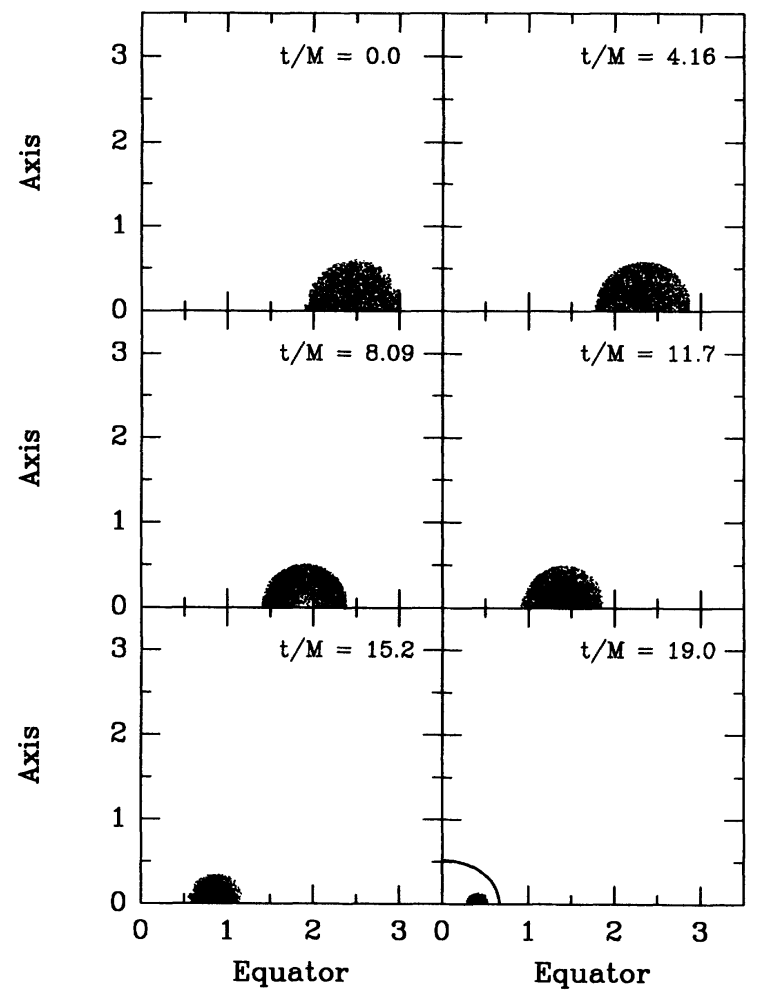

FIG. 4. Collapse of a nonequilibrium rotating toroid (case 10) to a Kerr black hole. The initial radius is $R_{s} / M=4.5$ and the $u_{\phi}$ countdown is 0.5. Each frame shows a $\phi=$ const slice of the axisymmetric solution. The apparent horizon is visible as a solid line in the final frame.

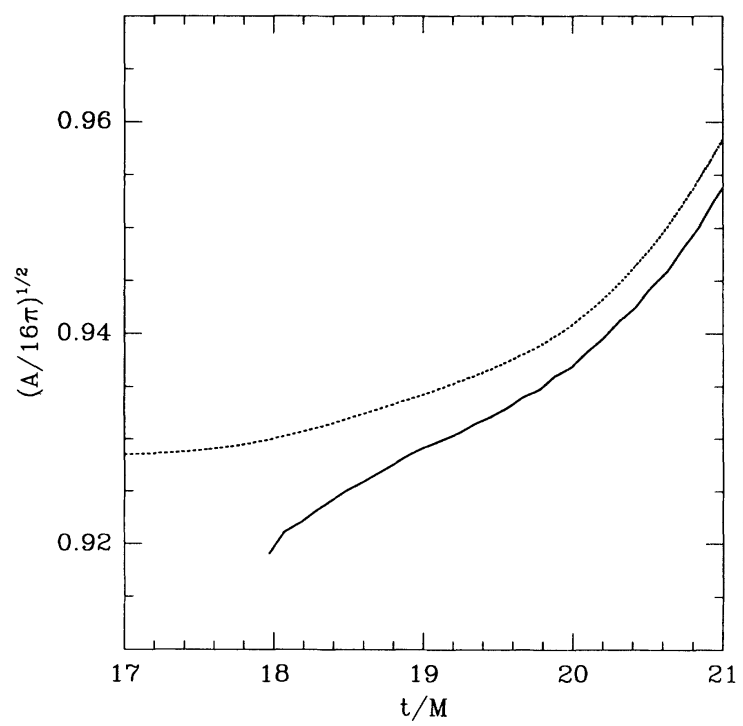

FIG. 5. Area of the apparent horizon as a function of time for the collapse shown in Fig. 4. The solid line shows the computed horizon area. The dotted line shows the theoretical value of the horizon area for a Kerr black hole with mass $M$ computed as a function of time during the simulation using Eq. (33) and angular momentum $J$ computed by Eq. (36). If mass were perfectly conserved numerically at the initial value $M=1$, the dotted line would be horizontal at $(\mathcal{A} / 16 \pi)^{1 / 2}=0.928$. 
and the area of the horizon approaches the Kerr value: $\mathcal{A} / 8 \pi M^{2}=1+\left(1-J^{2} / M^{4}\right)^{1 / 2}$. Once the black hole forms, maintaining resolution of the longitudinal part of the gravitational field becomes difficult because of throat stretching effects. Consequently the numerically calculated mass indicators start to diverge. This divergence is seen also in the apparent horizon area as a function of time, but we are still able to see agreement between the horizon area and Kerr prediction at the $1 \%$ level.

New methodology has been developed recently for locating event horizons in numerically generated spacetimes [20]. When applied to case 10, there is a clear indication that the event horizon is toroidal when it first forms. At late times as the event horizon and the apparent horizon converge, it becomes topologically spherical.

We increase the cut-down factor to 0.65 in case 11 and 0.72 in case 12 , thus increasing $J / M^{2}$ to 0.89 and 0.98 , respectively. In each case a Kerr hole forms, though the increased rotation does delay apparent horizon formation to coordinate times of roughly $24.5 M$ for case 11 and $30.5 \mathrm{M}$ for case 12 . For cut-down factors larger than 0.75 , no black hole is seen. Collapse still occurs, but is eventually halted by rotation. The final result is a smaller, apparently stable, equilibrium toroid. In case 14 $u_{\phi}$ is cut down by 0.90 , producing an angular momentum of $J / M^{2}=1.18$. The configuration collapses to a major radius of about 1.5 before stabilizing. This collapse is illustrated in Fig. 6. The Kerr rotation limit $J / M^{2}<1$ for black holes is obeyed, in accordance with cosmic cen-

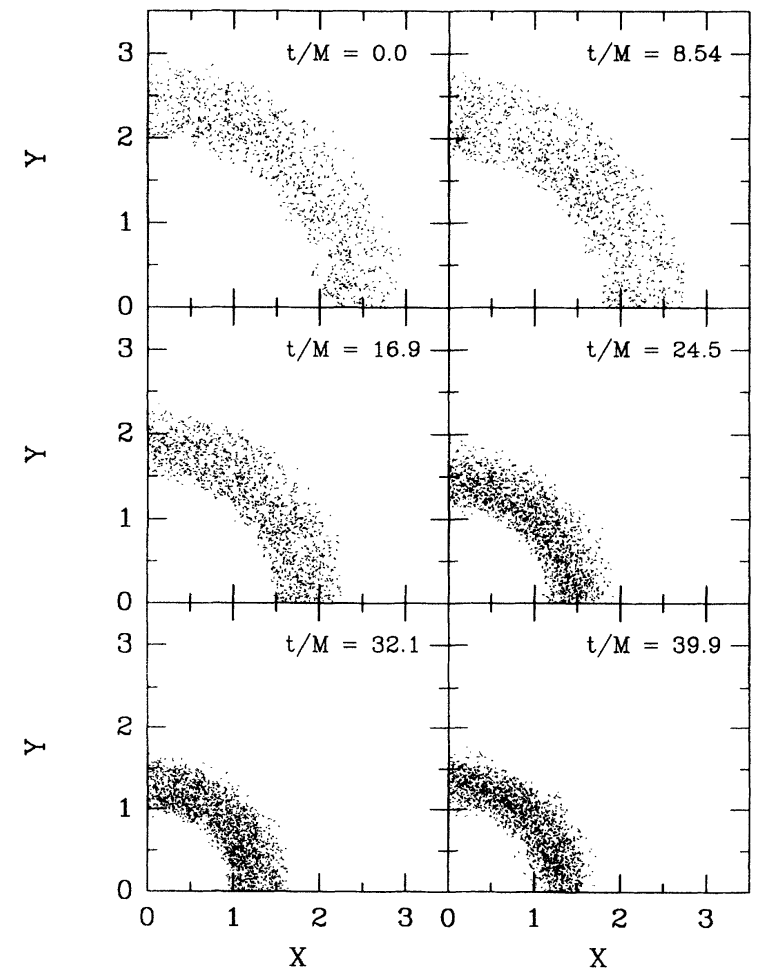

FIG. 6. Snapshots of the collapse of a nonequilibrium toroid with $J / M^{2}>1$ (case 14) to a new stable equilibrium configuration. Each frame shows the first quadrant of the equatorial plane.

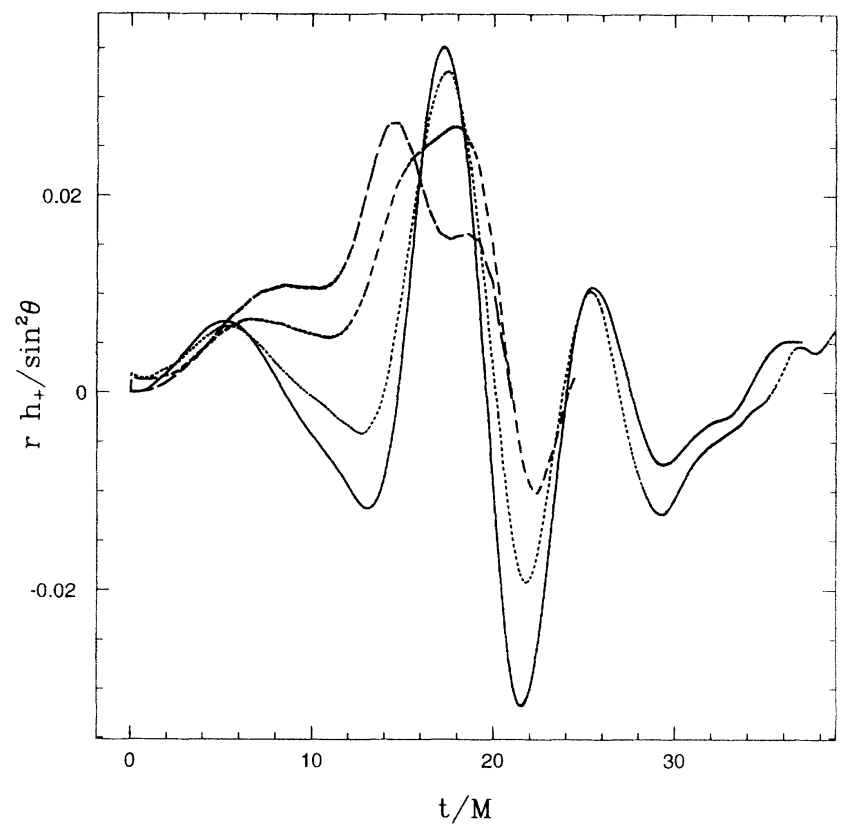

FIG. 7. Comparison of the $h_{+}$waveforms for four cases with initial radius $R_{s} / M=4.5$ and various values of the $u_{\phi}$ cut down factor. Case 15 (solid line) and case 14 (dotted line) have $J / M^{2}>1$. Case 10 (short dashed line) and case 11 (long dashed line) have $J / M^{2}<1$. We note that oscillations in the toroids' minor radius become less pronounced when they collapse, as reflected in $h_{+}$.

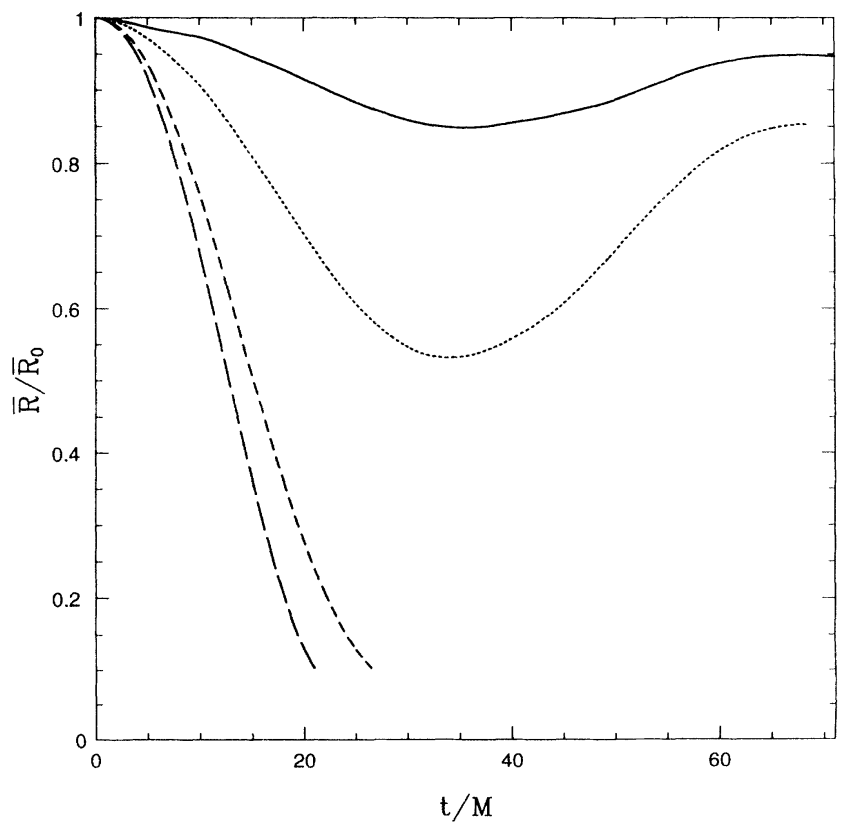

FIG. 8. Comparison of the mean radii $\bar{R}$ of the toroids as functions of time for four cases with initial $R_{s} / M=4.5$ and various values of the $u_{\phi}$ cut down factor. Case 15 (solid line) and case 14 (dotted line) have $J / M^{2}>1$, do not collapse to black holes, and show evidence of oscillations in $\bar{R}$. Case 10 (short dashed line) and case 11 (long dashed line) have $J / M^{2}<1$ and do collapse to Kerr holes. 
sorship. That is, unstable configurations with $J / M^{2}<1$ can collapse to black holes, while those with $J / M^{2}>1$ cannot form a singularity. Note that the toroidal configurations have $\delta$ function angular momentum distributions and there is no mechanism for the particles to transfer or radiate angular momentum in axisymmetry. Thus the numerical outcome of these collapse calculations is as expected; it is reassuring, however, to see this theoretical expectation reproduced numerically.

Finally, we contrast four of the nonequilibrium toroidal cases $(10,11,14$, and 15$)$ with $R_{s} / M=4.5$ initially and various $\phi$-velocity cut-down factors. In Fig. 7 we display the even-parity gravitational waveforms from these cases. The waveforms show similar shapes at early time because of the minor radius oscillations. The configurations that collapse substantially along the major radius, particularly those forming black holes, do not rebound as much in the minor radius direction so have smaller wave amplitudes in that phase of the waveforms. Odd-parity $\ell=3$ waves are emitted as well with smaller amplitudes but also apparently correlated with the minor radius oscillations. The energy radiated in gravitational waves was less than $0.1 \%$ of the total mass energy in all cases. In Fig. 8 we compare the behavior of the mean major-radius $\bar{R}$ for these evolutions. For cases 14 and 15 we see indications of oscillation and no black hole formation, whereas cases 10 and 11 (with $J / M^{2}<1$ ) clearly exhibit collapse.

\section{CONCLUSIONS}

We have presented a new numerical code that can solve Einstein's equations of general relativity for the evolution of rotating collisionless matter in axisymmetry. The code is used to diagnose the stability of relativistic equilibrium configurations. It is able to follow the collapse of unstable rotating configurations to Kerr black holes.

A number of interesting problems that can be addressed with this code are now open for investigation. For example, previous simulations [7] have studied the collapse of axisymmetric collisionless matter configurations without rotation. Compact clusters, both prolate and oblate, collapse to form black holes. However, sufficiently long prolate clusters collapse to spindle singularities without the formation of apparent horizons. These simulations suggest a possible violation of the cosmic censorship hypothesis. Later calculations of prolate collapse were carried out with equal numbers of corotating and counterrotating particles [8]. For sufficiently small rotation, the results were indistinguishable from the nonrotating cases to within the numerical accuracy. It would now be interesting to explore the same question but with net rotation in the collapsing cluster.

\section{ACKNOWLEDGMENTS}

This work was supported by National Science Foundation Grants Nos. AST 91-19475 and PHY 90-07834.
Computations were performed at the Cornell Center for Theory and Simulation in Science and Engineering, which is supported in part by the National Science Foundation, IBM Corporation, New York State, and the Cornell Research Institute.

\section{APPENDIX: REGULARITY OF VARIABLES}

Here we give the regular dependences of field quantities near the origin $[13,16]$. These are derived by assuming local flatness, expanding all functions in regular power series in Cartesian coordinates, and applying axisymmetry and equatorial plane symmetry conditions. In the case of the metric functions, the spatial gauge conditions are also applied. In what follows, the functions $f_{1}, f_{2}$, $f_{3}, g_{1}, g_{2}, g_{3}$, and $h_{1} \cdots h_{6}$, are assumed to be regular functions of $\rho^{2} \equiv r^{2} \sin ^{2} \theta$ and $z^{2}=r^{2} \sin ^{2} \theta$.

The shift vector, and any other vector field (e.g., $\hat{S}^{i}$ ), has an expansion of the form

$$
\begin{aligned}
& \beta^{r}=r\left(\sin ^{2} \theta f_{1}+\cos ^{2} \theta f_{2}\right), \\
& \beta^{\theta}=\cos \theta \sin \theta\left(f_{1}-f_{2}\right), \\
& \beta^{\phi}=-f_{3} .
\end{aligned}
$$

The metric variables are expanded as

$$
\begin{aligned}
A^{2} & =g_{1}+r^{2} \sin ^{2} \theta g_{2}, \\
B^{2} & =g_{1} \\
\xi & =r^{4} \cos \theta \sin ^{2} \theta \frac{g_{3}}{g_{1}}, \\
T^{2} & =1+r^{2} \sin ^{2} \theta \frac{g_{2}}{g_{1}}, \\
\eta & =\frac{1}{2} r^{2} \sin ^{2} \theta \frac{g_{2}}{g_{1}} .
\end{aligned}
$$

The extrinsic curvature, or another second-rank tensor field, obeys

$$
\begin{aligned}
& \hat{A}_{(1)(1)}= \sin ^{2} \theta h_{1}+\cos ^{2} \theta h_{2}+r^{2} \sin ^{4} \theta h_{3} \\
&+2 r^{2} \cos ^{2} \theta \sin ^{2} \theta h_{4}, \\
& \hat{A}_{(1)(2)}= \cos \theta \sin \theta\left(h_{1}-h_{2}+r^{2} \sin ^{2} \theta h_{3}\right. \\
&\left.+r^{2}\left(\cos ^{2} \theta-\sin ^{2} \theta\right) h_{4}\right), \\
& \hat{A}_{(1)(3)}=r^{2} \sin \theta\left(\sin ^{2} \theta h_{5}-\cos ^{2} \theta h_{6}\right) T, \\
& \hat{A}_{(2)(3)}=r^{2} \cos \theta \sin ^{2} \theta\left(h_{5}+h_{6}\right) T \\
& \hat{A}_{(3)(3)}=T^{2} h_{1} .
\end{aligned}
$$

Finally, the maximal slicing condition yields the relation (applicable to trace-free second-rank tensor fields)

$$
2 h_{1}+h_{2}+\left(\frac{h_{1} g_{2}}{g_{1}}+h_{3}\right) r^{2} \sin ^{2} \theta=0 .
$$


[1] L. Smarr, in Sources of Gravitational Radiation, edited by L. Smarr (Cambridge University Press, Cambridge, England, 1979).

[2] K. Eppley, in Sources of Gravitational Radiation [1].

[3] T. Nakamura, Prog. Theor. Phys. 65, 1876 (1981).

[4] R. F. Stark and T. Piran, Phys. Rev. Lett. 55, 891 (1985); T. Piran and R. F. Stark, in Dynamical Spacetimes and Numerical Relativity, edited by J. Centrella (Cambridge University Press, Cambridge, England, 1986); R. F. Stark and T. Piran, Comput. Phys. Rep. 5, 221 (1987).

[5] C. Evans, in Dynamical Spacetimes and Numerical Relativity [4]; A. M. Abrahams and C. R. Evans, Phys. Rev. D 46, R4117 (1992); Phys. Rev. Lett. 70, 2890 (1993).

[6] A. Abrahams, D. Bernstein, D. Hobill, E. Seidel, and L. Smarr, Phys. Rev. D 45, 3544 (1992).

[7] S. L. Shapiro and S. A. Teukolsky, Phys. Rev. Lett. 66, 994 (1991).

[8] S. L. Shapiro and S. A. Teukolsky, Phys. Rev. D 45, 2006 (1992).

[9] S. L. Shapiro and S. A. Teukolsky, Phys. Rev. D 45, 2739 (1992).

[10] S. L. Shapiro and S. A. Teukolsky, Astrophys. J. 419, 622 (1993).

[11] S. L. Shapiro and S. A. Teukolsky, Astrophys. J. 419, 636 (1993).

[12] S. L. Shapiro and S. A. Teukolsky, Phil. Trans. R. Soc.
A340, 365 (1992).

[13] J. M. Bardeen and T. Piran, Phys. Rep. 96, 205 (1983).

[14] J. M. Bardeen, in Gravitational Radiation, edited by N. Dereulle and T. Piran (North-Holland, Amsterdam, 1983).

[15] A. M. Abrahams and C. R. Evans, Phys. Rev. D 37, 318 (1988).

[16] C. R. Evans, Ph.D. thesis, University of Texas at Austin, 1984.

[17] H. O. Kreiss and J. Oliger, Methods for the Approximate Solution of Time Dependent Problems, GARP Publication Series No. 10 (World Meteorological Organization, Geneva, 1973).

[18] S. A. Teukolsky, Phys. Rev. D 26, 745 (1982).

[19] As noted in Sec. II C, because of the nonglobal nature of this gauge for axisymmetric, dynamical spacetimes, the asymptotic behavior of $\beta^{\phi}$ computed by the algorithm used in the evolution code differs from that of the same function determined for our stationary initial data. This difference has no physical significance as in axisymmetry one can make arbitrary angle-dependent rotations of the coordinate system at infinity.

[20] S. A. Hughes, C. R. Keeton, P. Walker, K. T. Walsh, S. L. Shapiro, and S. A. Teukolsky, Phys. Rev. D 49, 4004 (1994). 


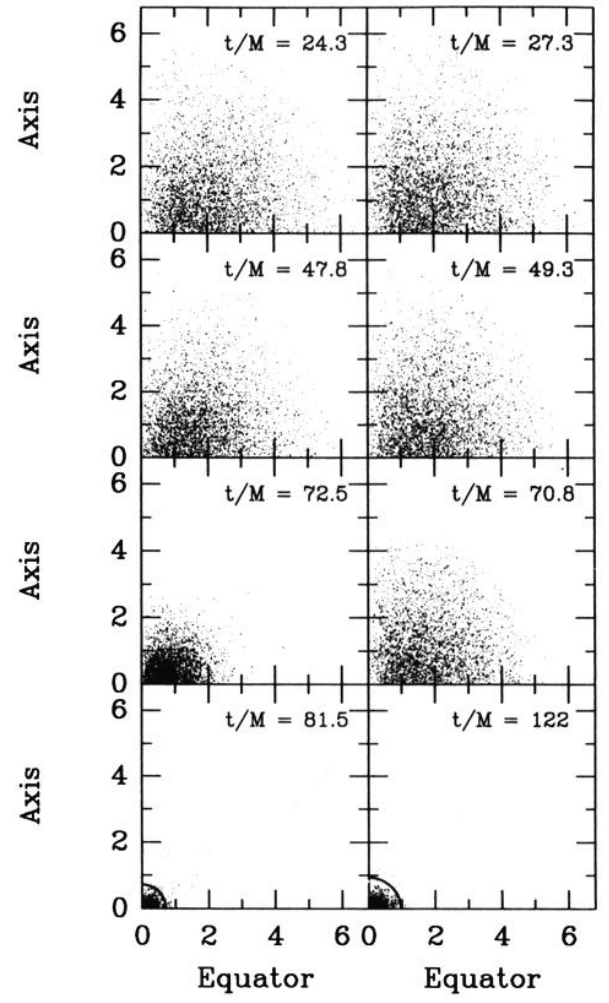

FIG. 2. Comparison of collapses of unstable polytropes. The left column shows snapshots from the collapse of a spherical nonrotating polytrope to a Schwarzschild black hole (case 2). This polytrope has equal numbers of corotating and counterrotating particles so that there is no net rotation. The right column shows the collapse of the analogous polytrope (case 5) constructed by making all the particles rotate with the same sense. While the evolution would be identical in Newtonian theory, the collapse of the rotating polytrope eventually leads to a Kerr black hole. An apparent horizon (solid line) forms in the nonrotating case at $t \sim 80 M$ and at $t \sim 120 M$ in the rotating case. Each frame shows a $\phi=$ const slice of the axisymmetric solution. 


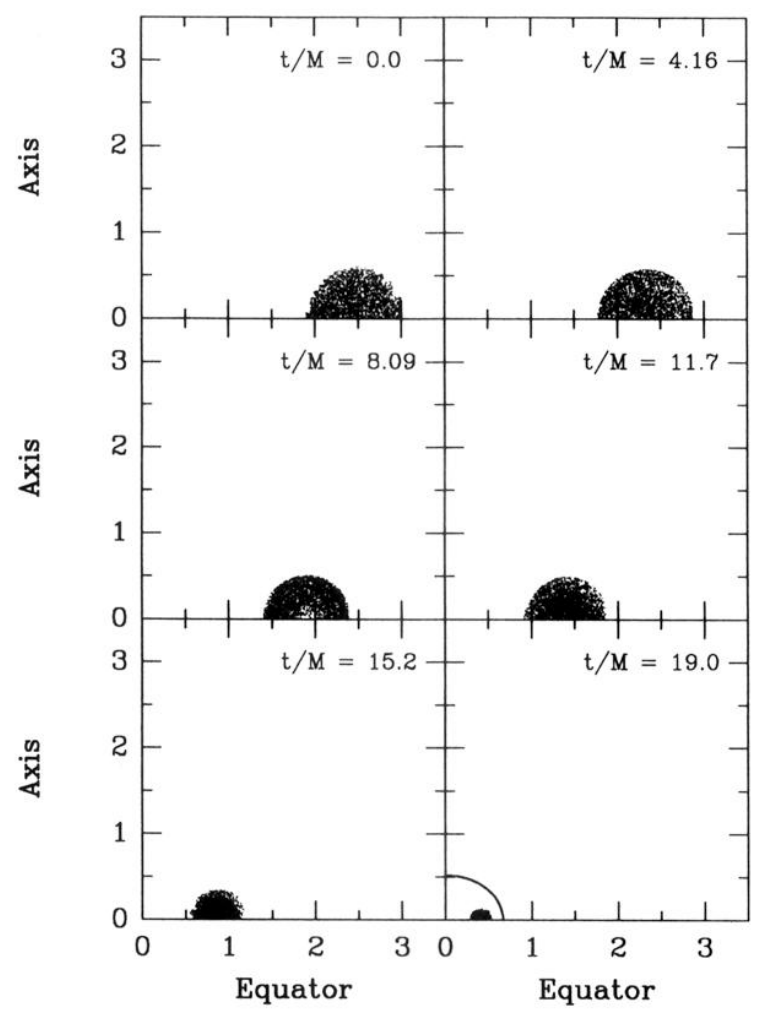

FIG. 4. Collapse of a nonequilibrium rotating toroid (case 10) to a Kerr black hole. The initial radius is $R_{s} / M=4.5$ and the $u_{\phi}$ countdown is 0.5 . Each frame shows a $\phi=$ const slice of the axisymmetric solution. The apparent horizon is visible as a solid line in the final frame. 


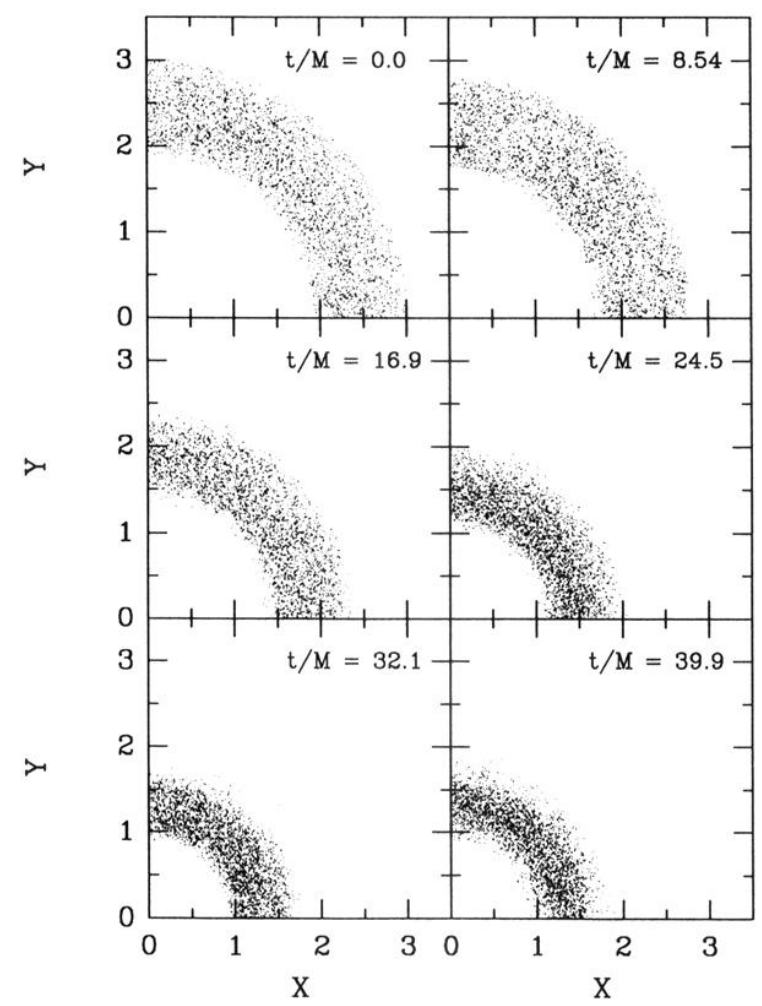

FIG. 6. Snapshots of the collapse of a nonequilibrium toroid with $J / M^{2}>1$ (case 14) to a new stable equilibrium configuration. Each frame shows the first quadrant of the equatorial plane. 